\title{
Continuous EEG Monitoring in Intensive Care Unit
}

\author{
Anuja Patil ${ }^{1}$ Sudhindra Vooturi ${ }^{1}$ Sita Jayalakshmi \\ ${ }^{1}$ Department of Neurology, Krishna Institute of Medical Sciences, \\ Secunderabad, Telangana, India
}

\begin{abstract}
Address for correspondence Sita Jayalakshmi, DM, Department of Neurology, Krishna Institute of Medical Sciences, Minister Road, 1-831/1, Secunderabad 500003, Telangana, India (e-mail: sita_js@hotmail.com;sita.js@gmail.com).
\end{abstract}

Int J Epilepsy 2018;5:62-67

\begin{abstract}
Keywords

- continuous EEG

- nonconvulsive status epilepticus

- nonconvulsive seizures

- neurocritical care unit

- encephalopathy

Trends of electroencephalogram (EEG) over 24 to 48 hours can help in prognostication in patients. Continuous electroencephalography (cEEG) allows for "real-time" bedside evaluation of cerebral function and can help to monitor patients in intensive care units. Lack of expertise in interpretation of the long-term EEG patterns and controversies in treatment implications have restricted the widespread use of this modality. This review summarizes the indications, techniques, duration, and pitfalls in CEEG monitoring. Compared with routine planned EEG, use of CEEG monitoring increases the sensitivity to detect nonconvulsive seizures (NCS) or nonconvulsive status epilepticus (NCSE) in unresponsive patients with no or subtle clinical signs of seizures. CEEG helps in reducing the overall intensive care unit (ICU) stay by timely detection of possible ischemic or ictal insults, alleviating the need for costlier imaging tests, and by precise drug adjustment in case of SE. However, standardization of the technical terms for wider applicability is needed. Analysis of automated computerized assays in seizure detection and their clinical role and addressing the technical aspects in long-term recordings should be evaluated; CEEG is gaining an important role in the multiparametric neuro-critical care units. Development of defined guidelines for the indications and application of CEEG, technological advances, and ongoing refinements are expected to enhance its utility in clinical practice.
\end{abstract}

\section{Introduction}

Patients in the intensive care units (ICUs) have to be monitored for various critical events such as seizures, elevated intracranial pressure, ischemia, infection, or alterations in mental status. Continuous electroencephalography (cEEG) allows for "real-time" bedside evaluation of cerebral function. Compared with routine planned EEG, it increases the sensitivity to detect nonconvulsive seizures (NCS) or nonconvulsive status epilepticus (NCSE) in unresponsive patients with no or subtle clinical signs of seizures. The dawn of computerized era has made long-term EEG monitoring using digitalized recording techniques and data transmission convenient. Trends of EEG over 24 to 48 hours can help in prognostication in comatose patients. The neuro-intensive care unit (NICU) of the European Society of Intensive Care Medicine (ESICM) has recommended guidelines with well-defined indications for cEEG monitoring in critically ill patients. ${ }^{1}$
Nonetheless, lack of expertise in interpretation of the longterm EEG patterns and controversies in treatment implications have restricted the widespread use of this modality. This review summarizes the indications, techniques, duration, and pitfalls in cEEG monitoring.

\section{Indications}

The indications for cEEG monitoring are summarized in - Table 1.2,3

\section{Nonconvulsive Seizures or Nonconvulsive Status Epilepticus}

Non convulsive seizures (NCS) or NCSE is common in critically ill patients and associated with poor neurological outcome and significant mortality. Use of cEEG monitoring has shown that NCS occurs in $48 \%$ and NCSE in $14 \%$ of patients

\section{received}

October 16, 2018

accepted after revision

May 17, 2019
DOI https://doi.org/

10.1055/s-0039-1693079

ISSN 2213-6320.
Copyright @2018 Indian Epilepsy Society
License terms

() (1) $\Theta \circledast$ 
Table 1 Indications for continuous EEG monitoring in critically ill patients 2,3

\begin{tabular}{|c|c|}
\hline S. No. & Clinical indication for CEEG monitoring \\
\hline 1. & $\begin{array}{l}\text { Detection of nonconvulsive seizures/nonconvul- } \\
\text { sive status epilepticus, and monitoring effect of } \\
\text { antiepileptic therapy } \\
\text { a. After convulsive status epilepticus } \\
\text { b. Unexplained alteration of mental status } \\
\text { c. Fluctuating mental status }\end{array}$ \\
\hline 2. & $\begin{array}{l}\text { Characterization of paroxysmal events } \\
\text { a. Abnormal movements such as myoclonus } \\
\text { b. Sustained posturing, head or eye deviation, } \\
\text { pupillary hippus } \\
\text { c. Paroxysmal autonomic spells such as } \\
\text { tachycardia }\end{array}$ \\
\hline 3. & Monitoring the depth and trends of sedation \\
\hline 4. & Grading the severity of encephalopathy \\
\hline 5. & $\begin{array}{l}\text { Detection of cerebral ischemia } \\
\text { a. After subarachnoid hemorrhage } \\
\text { b. During or after vascular interventional radio- } \\
\text { logical procedures } \\
\text { c. Patients with hemodynamic instability }\end{array}$ \\
\hline 6. & $\begin{array}{l}\text { Prognostication } \\
\text { a. Hypoxic-ischemic encephalopathy } \\
\text { b. Post cardiac arrest }\end{array}$ \\
\hline 7. & $\begin{array}{l}\text { During therapeutic hypothermia and within } 24 \\
\text { hours of rewarming }\end{array}$ \\
\hline 8. & Brain death \\
\hline
\end{tabular}

Abbreviation: cEEG, continuous electroencephalography.

admitted to ICU with seizures. ${ }^{4}$ It can occur in patients without prior seizures with acute neurological conditions such as stroke, CNS infections, traumatic brain injuries, post neurosurgical procedures; or it may complicate systemic disorders such as sepsis, metabolic encephalopathies, withdrawal from alcohol, opiates, benzodiazepines or is associated with use of antibiotics such as cefepime, clarithromycin, or fluroqunolones. ${ }^{5,6}$ Both NCS and NCSE may exist with subtle clinical signs, or as mere confusion, agitation, aphasia, or behavioral alteration even without preceding seizures. Clinical recognition may be confounded by the common use of sedation, anesthetics, or neuromuscular blocking agents in patients in ICU, thereby highlighting the role of CEEG monitoring in such cases. Specific EEG patterns are significantly associated with seizures ( $\bullet$ Fig. 1) either NCS/NCSE, such as generalized periodic discharges (GPDs), lateralized periodic discharges (LPDs), or bilateral independent periodic discharges (BIPDs), lateralized rhythmic delta activity (LRDA), or as burst suppression pattern with intermittent ictal discharges against a "flat" background. Monitoring with CEEG helps not only to detect seizure, but also to assess efficacy of antiepileptic and anesthetic treatment during SE with suppression of these patterns. Although introduction or intensification of antiepileptic therapy with early detection of NCS/NCSE on cEEG is expected to improve the neurological state, the underlying etiology and comorbidities remain significant predictors of outcome.

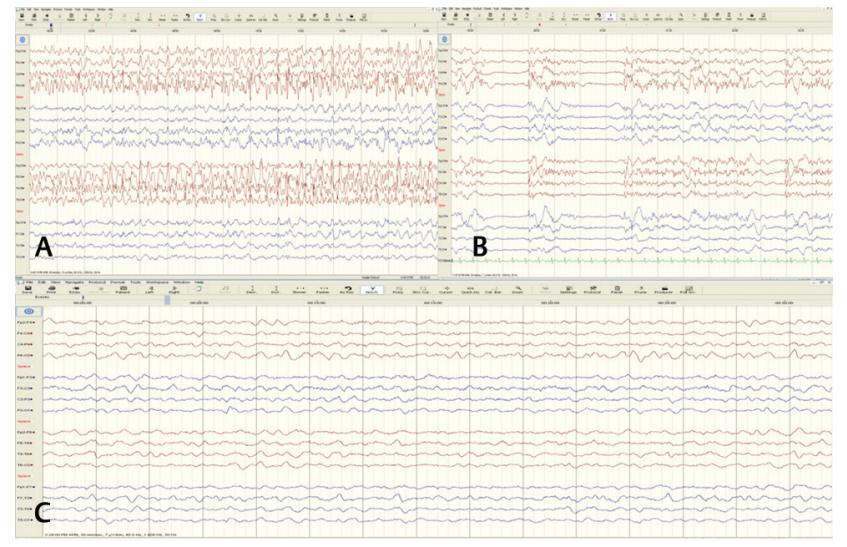

Fig. 1 (A) EEG of a 13-year-old girl with convulsive status epilepticus showing rhythmic spike discharges along the right temporal region spreading to right hemispheres, suggestive of right temporal epileptogenic focus. (B) After antiepileptic therapy, she continued to have subtle SE with EEG showing burst suppression pattern. (C) After initiation of anesthetic coma, the patterns resolved into diffuse background theta range slowing. EEG, electroencephalography.

\section{Characterization of Paroxysmal Events}

In critically ill and comatose patients, subtle paroxysmal movements may raise concern for ongoing seizures such as myoclonus, nystagmus, gaze deviation, and brief facial twitching. In cases that are otherwise unexplained, such as paroxysmal tachycardia or episodic posturing, it is essential to evaluate presence of an ictal correlate. A long-term video EEG record may help delineate occurrence of epileptiform discharges associated with these movements. If myogenic artifacts obscure the EEG findings, use of a short-acting neuromuscular blocking agent in patients on ventilatory support may help to recognize presence of any EEG abnormality.

\section{Grading the Severity of Encephalopathy and Prognostication}

The EEG patterns correlate with severity of encephalopathy associated with sepsis or other metabolic conditions, ranging from mild slowing in theta ( $>4$ but $<8 \mathrm{~Hz}$ ) to intermittent delta range $(\leq 4 \mathrm{~Hz})$ to rhythmic and later continuous and arrhythmic, then occurrence of triphasic, and ultimately to burst suppression pattern. There is linear correlation of worsening of EEG patterns with increasing mortality due to multiorgan failure. cEEG can help in assessing the course and effect of therapy. ${ }^{2}$ The predictors of poor outcome in patients with encephalopathy include age more than 65 years, anoxicischemic etiology, background EEG suppression, and lack of EEG reactivity.

The EEG grading may help to understand the severity of encephalopathy (-Fig. 2) and help in the prognosis ${ }^{7}$ :

Grade 1: Background is $\alpha$ rhythm with or without scattered theta range activity ( $\mathbf{- \text { Fig. }} \mathbf{2 A}$ ).

Grade 2: Background of theta intermixed with some $\alpha$ or delta range activity ( - Fig. $\mathbf{2 B}$ ).

Grade 3: Background of continuous polymorphic delta with little faster frequencies. Reactivity to external stimuli and variability present ( $\boldsymbol{- \text { Fig. }} \mathbf{2 C}$ ). 


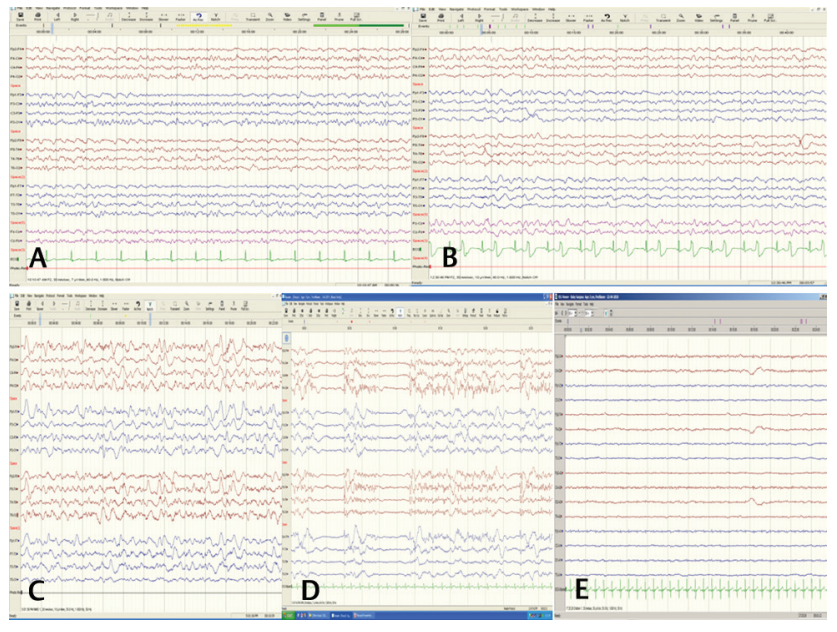

Fig. 2 Grading of severity of encephalopathy.

Grade 4: Invariant delta of small amplitude of $<100 \mu \mathrm{V}$ and unresponsive or suppression burst pattern (-Fig. 2D)

Grade 5: A nearly flat or isoelectric tracing ( - Fig. 2E).

\section{Detection of Cerebral Ischemia}

Delayed cerebral ischemia (DCI) is a grave complication in patients with subarachnoid hemorrhage and is known to occur in approximately $20 \%$ to $30 \%$ of cases. Clinical and radiographic techniques often are able to detect these changes with small therapeutic window. Changes in cerebral perfusion manifest on EEG recording in the form of regional attenuation of fast frequencies and increase in slow activity. Monitoring with cEEG is currently recommended for detection of DCI after subarachnoid hemorrhage. ${ }^{1}$ There is a drop in sensorium with clinical deterioration due to vasospasm-induced cerebral ischemia, theoretically electrographic changes can be detected before structural damage can be seen on imaging, thereby providing an opportunity to intervene early. ${ }^{8}$ Various quantitative EEG parameters (qEEG)-composite $\alpha$ index, relative $\alpha$ variability (RAV), total power, and $\alpha /$ delta ratio (ADR) have been described to be useful to detect DCI 24 to 48 hours prior to other diagnostic tools. ${ }^{9}$ Apart from several retrospective studies supporting the role of cEEG, recent prospective study ${ }^{10}$ suggested that new or worsening ADR trends, RAV trends, focal slowing, epileptiform discharges, and rhythmic or periodic EEG patterns can predict DCI after SAH with excellent sensitivity and high specificity. To predict an occurrence of DCI before clinical symptoms, the number of patients needed to monitor varied from 3 to 7 .

\section{Prognostication}

In comatose patients, especially in anoxic-ischemic encephalopathy post cardiac arrest, or in traumatic brain injury patients and ischemic or hemorrhagic stroke patients, EEG may aid as a prognostic tool. Certain EEG patterns have been identified as indicators of poor outcome such as complete background suppression, a burst suppression with generalized periodic epileptiform discharges within bursts, or generalized periodic epileptiform discharges against a near flat background ${ }^{4,11}$ Reactivity to external stimuli in the form of change in amplitude or frequency in background rhythms indicates a better chance of recovery together with other clinical parameters. This should be determined with various stimuli such as loud sounds, response to pain, and passive eye opening. Worsening EEG patterns are associated with mortality as studied in sepsis-associated encephalopathy (- Table 2). ${ }^{2}$ Further studies are required, however, to determine the EEG parameters and their predictive roles.

\section{Technical and Operating Guidelines}

\section{EEG Electrodes and Montages}

Although a standard EEG montage with 21 electrodes with $10-20$ system is recommended, ${ }^{1}$ in the ICU settings it may not be feasible due to lack of expertise or in applying all scalp electrodes in case of neurosurgical or trauma patients. A more simplified electrode placement method may be used in emergent situations of the neuro-critical care unit. Three different montages-double diamond, circumferential, and $\mathrm{Cz}$ referential-using easy placement of 7 electrodes (Fp1, $\mathrm{Fp} 2, \mathrm{~T} 3, \mathrm{~T} 4, \mathrm{O} 1, \mathrm{O} 2$, and $\mathrm{Cz}$ ) based on anatomical landmarks were shown to be almost $93 \%$ sensitive for seizure detection. ${ }^{12}$ Standard collodion applied disk electrodes are preferable due to easy applicability. However, these may fail during long-term EEG monitoring. Subdermal wire electrodes secured with collodion or any sealant are used at some centers. ${ }^{13}$ Video EEG recording is preferred so as to correlate the EEG discharges with any paroxysmal movements seen.

Continuous raw EEG data may be laborious to interpret. Automated displays using color density spectral array (CDSA) and amplitude-integrated EEG compress the EEG into trends of changes in frequencies and voltage against time. These displays make it feasible for non-neurologists and paramedical staff to track the changes in EEG trends and seizure detection bedside.

\section{Duration of Monitoring}

Almost $50 \%$ of NCS have been shown to be detected within first 60 min of EEG recording. ${ }^{14}$ Almost 95\% noncomatose patients had their first seizure detected on CEEG within first 24 hours of initiation of the monitoring, while only $80 \%$ of comatose patients did so. Recording for at least of 24 hours is recommended in critically ill patients. ${ }^{14}$ Routinely duration for monitoring patients with cEEG is dictated by the indication, probability of seizures, and likelihood of further deterioration in clinical course. Patients who are under

Table 2 Graded mortality associated with EEG patterns in sepsis-associated encephalopathy ${ }^{2}$

\begin{tabular}{|l|l|}
\hline EEG pattern & Percentage mortality \\
\hline Theta & 40 \\
\hline Delta & 48 \\
\hline Triphasic waves & 53 \\
\hline Burst suppression & 70 \\
\hline
\end{tabular}

Abbreviation: EEG, electroencephalography. 
sedation or already treated with antiepileptics may need prolonged monitoring to detect occurrence of NCS. Lack of any epileptiform discharges in the initial ( $<2$ hours) recording was suggestive of low $(<5 \%)$ chance of seizures in the next 72 hours. Brief 30-min EEGs repeated serially were also shown to have similar clinical yield in patients post cardiac arrest as monitored with cEEG. ${ }^{15}$ Longer duration up to several days may be required in setting of detection of DCI in patients with SAH or ischemic strokes.

\section{Significant EEG Patterns}

\section{Periodic Discharges}

Periodic discharges (PDs) including lateralized (LPDs) or generalized (GPDs) are associated with worse outcomes in post hypoxic encephalopathy or as marker of SE, but can be seen in other conditions such as acute infarct, herpes encephalitis, autoimmune encephalitis, and Creutzfeldt-Jakob disease (-Figs. 3 and 4 ).

\section{Triphasic Waves}

These are high-amplitude (200-300 $\mu \mathrm{V})$, positive sharp transients preceded by short duration, small-amplitude negative sharp waves followed by a long-duration negative slow wave, with fronto-occipital lag of 25 to 140 milliseconds. They may be mono-, di- or quadric-phasic and may occur as short or long paroxysms, or repeat at 2 to $4 \mathrm{c} / \mathrm{s}$ (pseudoparoxysmal). They are typically seen in and highly specific for hepatic encephalopathy but may also occur in uremic encephalopathy, anoxia, hyperosmolar state, and metabolic disturbances such as hypoglycemia, hyponatremia, hypercalcemia, and hyperthyroidism ${ }^{16}$ ( - Fig. 5A-C)

\section{Ictal-Interictal Continuum}

Several of the above-described patterns are not clearly ictal or interictal, especially in critically ill patients. GPDs or LPDs are seen in approximately $12 \%$ to $14 \%$ of cEEG recordings. ${ }^{17}$

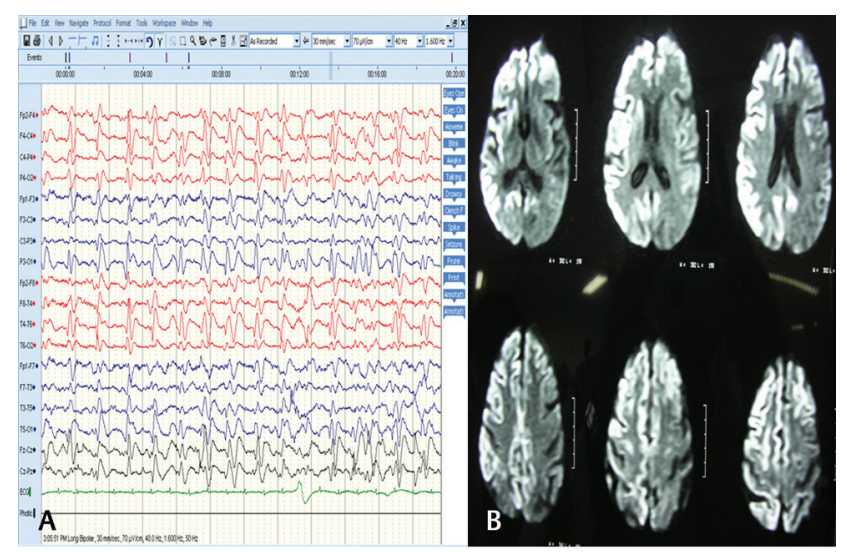

Fig. 3 A 69-year-old lady with progressive behavioral disturbances since 1 month and new onset generalized seizures with occasional myoclonus developed altered sensorium for 3 days. (A) EEG showing periodic $1 \mathrm{~Hz}$ generalized sharp and triphasic discharges. (B) MRI showing cortical ribboning on DW sequences suggestive of Creutzfeldt-Jakob disease. EEG, electroencephalography; MRI, magnetic resonance imaging.

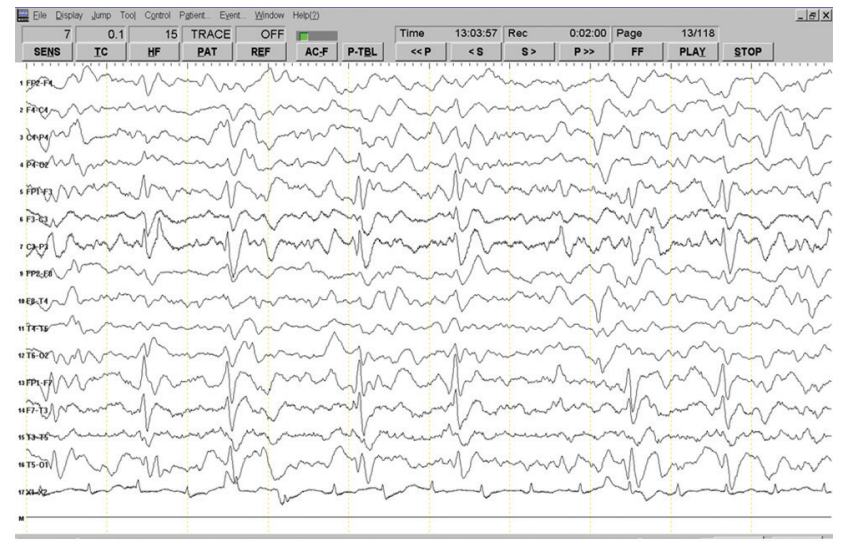

Fig. 4 Bilateral periodic discharges seen in a 27-year-old man diagnosed with herpes encephalitis.

Presence of these discharges not only supports NCS/NCSE in the acute settings but also predicts future risk. Patients with LPDs are at almost seven times increased risk of developing epilepsy..$^{18}$ Lateralized rhythmic delta activity has a similar significance and is associated with high risk of acute, especially nonconvulsive seizures. ${ }^{19}$ Clinical correlates may not always be discernible in comatose patients, thereby making the addition of antiepileptics or sedation controversial. A trial of benzodiazepines may be diagnostic if it resolves the EEG patterns and at the same time leads to clinical improvement. However, this may not always be conclusive and carries potential sedative effect, which may not be acceptable in all clinical settings especially with hepatic or renal dysfunction. A nonsedating antiepileptic drug trial may serve as a better alternative. ${ }^{20} \mathrm{~A}$ thorough clinical evaluation of the probable etiology, prior ictal rhythms, and nature of evolution of current ambiguous EEG patterns along with proper imaging (magnetic resonance imaging [MRI]-diffusion weighted imaging [DWI] and perfusion studies) and biochemical parameters (serum neuron-specific enolase NSE) may help in the management $\mathrm{t}^{20}$ (-Fig. 6 ).

\section{Limitations}

Few of the practical limitations of cEEG include ${ }^{2,3}$ the following:

- The vast data provided by CEEG are labor intensive both for technologists and clinicians.

- Quantitative EEG algorithms with compressed spectral arrays can prove helpful but need validation for clinical application.

- The compatibility of routinely used electrodes may interfere with other investigations such as MRI.

- For patients on ventilatory support, pacemakers, air beds, and infusion pumps, or with insistent movements such as chewing and myoclonic jerking may pose additional artifacts during recordings. Video-EEG recording may help recognizing theseartifacts.

- There is no standard nomenclature or classification into ictal-interictal categories of commonly seen rhythmic or periodic patterns in critically ill patients, creating interoberver bias and difficulty in analyzing records. 


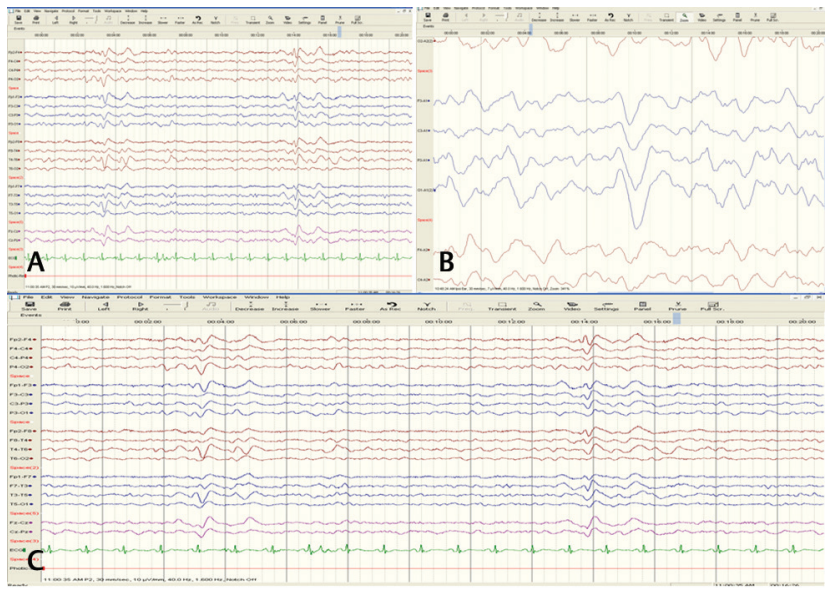

Fig. 5 Triphasic waves seen in (A) patient with hepatic failure, (B) Hashimoto encephalitis, (C) the initial slow and sharp negative wave followed by large amplitude positive sharp wave followed by long-duration negative wave.

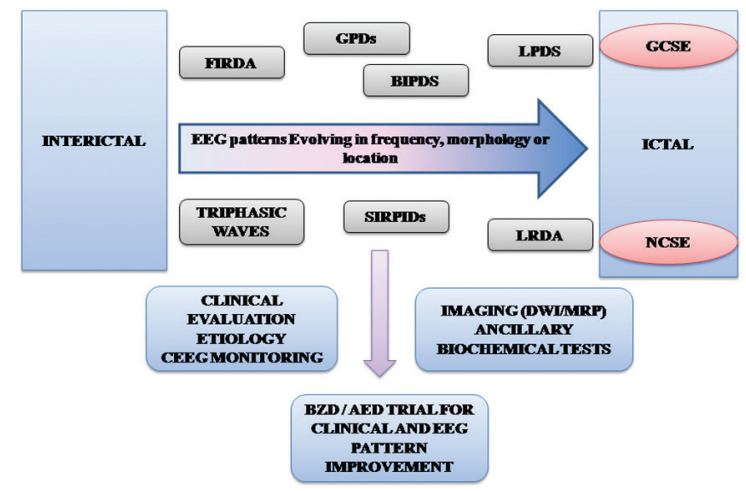

Fig. 6 The ictal-interictal continuum. EEG patterns seen during cEEG should be interpreted with caution and always with the clinical context. FIRDA, frontal intermittent rhythmic delta activity; GPDs, generalized periodic discharges; BIPDs, bilaterally independent periodic discharges; SIRPDs, stimulus-induced rhythmic, periodic, or ictal discharges; LPDs, lateralized periodic discharges; LRDA, lateralized rhythmic delta activity; GCSE, generalized convulsive status epilepticus; NCSE, nonconvulsive status epilepticus; DWI, diffusion-weighted imaging; MRP, MRI perfusion study; BZD, benzodiazepine; AED, antiepileptic drugs.

- At times, detection of ambiguous patterns may subject the patients for further expensive evaluations and prolonged sedation or antiepileptic treatments.

- It remains unclear whether aggressive treatment of intermittent NCS alters the final outcome. Thus, caution must be exercised not only in interpretation of the vast data, but its clinical implications as well.

Though current literature on the relative cost-effectiveness of cEEG in intensive care units is lacking, with increasing availability of these technical amenities at lower cost over past few years, it is promising for widespread use in practice. When implemented, cEEG may help reduce the overall ICU stay by timely detection of possible ischemic or ictal insults, alleviating the need for costlier imaging tests and by precise drug adjustment in case of SE.

\section{Future Research}

Many unresolved questions in cEEG monitoring include the following:

- EEG patterns that should be chased therapeutically,

- Standardization of the technical terms for wider applicability,

- Analyzing the automated computerized assays in seizure detection and their clinical role,

- Addressing the technical aspects in long-term recordings.

\section{Conclusion}

Use of cEEG is gaining an important role in the multiparametric neurocritical care units. Development of defined guidelines for the indications and application of CEEG, technological advances, and ongoing refinements are expected to enhance its utility in practice.

\section{Author Contribution}

Dr. Anuja Patil-writing manuscript and literature review,

Dr. Sita Jayalakshmi-critical revision of the manuscript,

Dr. Sudhindra Vooturi-review of manuscript

\section{Conflict of Interest}

None declared.

\section{References}

1 Claassen J, Taccone FS, Horn P, Holtkamp M, Stocchetti N, Oddo $\mathrm{M}$; Neurointensive Care Section of the European Society of Intensive Care Medicine. Recommendations on the use of EEG monitoring in critically ill patients: consensus statement from the neurointensive care section of the ESICM. Intensive Care Med 2013;39(8):1337-1351

2 Young GB, Mantia J. Continuous EEG monitoring in the intensive care unit. Handb Clin Neurol 2017;140:107-116

3 Hirsch LJ. Continuous EEG monitoring in the intensive care unit: an overview. J Clin Neurophysiol 2004;21(5):332-340

4 DeLorenzo RJ, Waterhouse EJ, Towne AR, et al. Persistent nonconvulsive status epilepticus after the control of convulsive status epilepticus. Epilepsia 1998;39(8):833-840

5 Kurtz P, Gaspard N, Wahl AS, et al. Continuous electroencephalography in a surgical intensive care unit. Intensive Care Med 2014;40(2):228-234

6 Anuhya V, Kunder SK, Madhyastha S, et al. Looking beyond the obvious: cefepime-induced nonconvulsive status epilepticus. J Pharmacol Pharmacother 2017;8(3):145-147

7 Synek VM. Prognostically important EEG coma patterns in diffuse anoxic and traumatic encephalopathies in adults. J Clin Neurophysiol 1988;5(2):161-174

8 Gaspard N. Current clinical evidence supporting the use of continuous EEG monitoring for delayed cerebral ischemia detection. J Clin Neurophysiol 2016;33(3):211-216

9 Vespa PM, Nuwer MR, Juhász C, et al. Early detection of vasospasm after acute subarachnoid hemorrhage using continuous EEG ICU monitoring. Electroencephalogr Clin Neurophysiol 1997;103(6):607-615

10 Rosenthal ES, Biswal S, Zafar SF, et al. Continuous electroencephalography predicts delayed cerebral ischemia after subarachnoid hemorrhage: a prospective study of diagnostic accuracy. Ann Neurol 2018;83(5):958-969 
11 Young GB. The EEG in coma. J Clin Neurophysiol 2000;17(5):473-485

12 Karakis I, Montouris GD, Otis JA, et al. A quick and reliable EEG montage for the detection of seizures in the critical care setting. J Clin Neurophysiol 2010;27(2):100-105

13 Claassen J, Mayer SA, Kowalski RG, Emerson RG, Hirsch LJ. Detection of electrographic seizures with continuous EEG monitoring in critically ill patients. Neurology 2004;62(10):1743-1748

14 Herman ST, Abend NS, Bleck TP, et al; Critical Care Continuous EEG Task Force of the American Clinical Neurophysiology Society. Consensus statement on continuous EEG in critically ill adults and children, part I: indications. J Clin Neurophysiol 2015;32(2):87-95

15 Crepeau AZ, Fugate JE, Mandrekar J, et al. Value analysis of continuous EEG in patients during therapeutic hypothermia after cardiac arrest. Resuscitation 2014;85(6):785-789

16 Bahamon-Dussan JE, Celesia GG, Grigg-Damberger MM. Prognostic significance of EEG triphasic waves in patients with altered state of consciousness. J Clin Neurophysiol 1989;6(4):313-319

17 Rodriguez Ruiz A, Vlachy J, Lee JW, et al; Critical Care EEG Monitoring Research Consortium. Association of periodic and rhythmic electroencephalographic patterns with seizures in critically ill patients. JAMA Neurol 2017;74(2):181-188

18 Punia V, Bena J, Krishnan B, Newey C, Hantus S. New onset epilepsy among patients with periodic discharges on continuous electroencephalographic monitoring. Epilepsia 2018;59(8):1612-1620

19 Gaspard N, Manganas L, Rampal N, Petroff OA, Hirsch LJ. Similarity of lateralized rhythmic delta activity to periodic lateralized epileptiform discharges in critically ill patients. JAMA Neurol 2013;70(10):1288-1295

20 Sivaraju A, Gilmore EJ. Understanding and managing the ictal-interictal continuum in neurocritical care. Curr Treat Options Neurol 2016;18(2):8 